Ciencias de los Alimentos/Food Sciences

Cienc Tecn UTEQ (2018) 11(2) p 47-56 ISSN 1390-4051; e-ISSN 1390-4043 CC) (i) \&)( ) DOI: https://doi.org/10.18779/cyt.v11i2.252

\title{
Análisis de la sección de destilación de una destilería de aguardiente de caña aplicando un simulador comercial
}

\author{
Analysis of the distillation section of a distillery of cane spirit applying a commercial simulator \\ Lauzurique Guerra Yeney ${ }^{1},{ }^{\circ}$ Pérez Ones Osney ${ }^{1}$, Zumalacárregui de Cárdenas Lourdes ${ }^{1}$, Rodríguez Cabrera Zuanchyt ${ }^{1}$ \\ ${ }^{1}$ Universidad Tecnológica de La Habana, Facultad de Ingeniería Química, "José Antonio Echeverría”. Departamento de \\ Ingeniería Química. Grupo de Análisis de Procesos. CUJAE. Av. 114 N. 11901 el Ciclovía y Rotonda. Marianao. \\ La Habana, Cuba.Tel.53-7-266-3208,Fax 53-7-267-2964. osney@quimica.cujae.edu.cu
}

Rec.: 24.07.2018. Acept.: 01.12.2018 Publicado el 30 de diciembre de 2018

\section{Resumen}

$E_{y}^{1}$ trabajo presenta una solución de los balances de masa y energía para el sistema integrado por la columna destiladora de aguardiente de caña, el sistema calientavinoscondensadores, la torre de enfriamiento y el generador de vapor, para una destilería de aguardiente de caña cubana. El proceso se modeló en el simulador comercial HYSYS v3.2 y los resultados para el sistema calientavinos - condensadores se compararon con los balances realizados manualmente utilizando el diagrama de entalpía - composición para el sistema etanol - agua, representativo del equilibrio líquido vapor a $101,3 \mathrm{kPa}$, con el fin de verificar la validez del modelo de simulación. Los resultados difieren en menos del 5\% por lo que el modelo reproduce el comportamiento del sistema. Con el modelo de simulación se analizó la influencia de la variación del flujo másico de batición y de la temperatura del vino a la salida del calientavinos sobre el flujo y composición del aguardiente producido, proponiendo como mejores condiciones de operación el trabajo al mayor flujo de batición posible $\left(40000 \mathrm{~kg} / \mathrm{h}\right.$ ) y calentando el vino hasta $90{ }^{\circ} \mathrm{C}$ en el calientavinos. Se verificó el desempeño del generador de vapor que alimenta a la columna de destilación, comprobando la posibilidad de recuperación del 19\% de la energía calorífica que se cede actualmente con los gases de combustión.

Palabras clave: aguardiente, condensador, destilación, generadores de vapor.

\begin{abstract}
$\mathrm{T}$ he present work offers a solution of mass and energy balances for a Cuban distillery, for a system formed by a rum column distillation, wine heater-condensers, a cooling tower and a boiler. The process was modeling in commercial simulator HYSYS v3.2 and the results were compared with an enthalpy diagram - composition for the ethanol - water system, representative of the vapor-liquid equilibrium at $101.3 \mathrm{kPa}$ in order to verify the kindness of the simulation model. Results differs less than $5 \%$, so the model reproduce the process behavior. Using the simulation model, mass flow and outlet wine's temperature influence on the spirit mass and its composition were analyzed. The best operating conditions were selected as $40000 \mathrm{~kg} / \mathrm{h}$ of feed stream and $90{ }^{\circ} \mathrm{C}$ at the outlet wine's heater. The behavior of the boiler that feed the distillation column was checked. Recovering $19 \%$ of the combustion gasses' heat is possible.
\end{abstract}

Keywords: boiler, condenser, distillation, spirit. 


\section{Introducción}

$\mathrm{E}$ espeso líquido marrón (melaza) que queda luego de la extracción del azúcar de caña puede destilarse para producir una estimulante bebida alcohólica, universalmente conocida por ron (Cardona et al., 2005; Fernández, 2016; Oliveira et al., 2016). Al hablar del ron cubano, no existe duda alguna de la obligación de remitirse al aguardiente procedente de la caña de azúcar, que definitivamente es quien le da su identidad insustituible. Mediante el proceso de destilación continua, se extraen los componentes volátiles del vino fermentado a través del intercambio de calor y masa en cada uno de los platos, empleando como fuerza motriz el vapor proveniente del generador de vapor (Pérez et al., 2010; Blanco, 2011).

Con la crisis en el sector energético en la década del 70, se desarrollaron estudios con la finalidad de encontrar alternativas para aumentar la eficiencia de los procesos de destilación, que hoy continúan siendo de interés tanto en el ámbito industrial como en el universitario (Pérez et al., 2011; Duro y Morilla, 2016). Cuba no está ajena a este interés.

La destilería que se analizó cuenta con un sistema para la producción de aguardiente de caña con una capacidad de $65000 \mathrm{~L} / \mathrm{d}$, en el cual la zona de agotamiento está construida de acero inoxidable y el resto de los equipos principales de cobre. El calientavinos con que se contaba en la destilería no garantizaba una temperatura superior a $56^{\circ} \mathrm{C}$ producto de su capacidad, incrustación y la existencia de múltiples salideros por los tubos. Estos salideros no podían eliminarse ya que se habían colocado tapones de acero al carbono que, al ser soldados al acero inoxidable con electrodos, formaron óxidos que no permitían nuevas soldaduras. Esto trajo afectaciones continuas a la calidad y al volumen de producción, ya que existía presencia de batición en el producto. Esta batición lo inhabilitaba para su añejamiento y comercialización por imprimirle color amarillo, olor y sabor putrefacto.

Para suplir los problemas existentes se buscaron alternativas, ya que elevar la calidad de la producción de aguardiente era imprescindible para mantener la estable y alta calidad físico-química y sensorial de los rones "Havana Club". Una alternativa consistió en instalar en la columna destiladora de aguardiente los condensadores que se encontraban en una columna rectificadora en desuso, lo que conllevó a modificaciones en el proceso. Buscar otras condiciones de operación que permitieran incrementar la cantidad del aguardiente producido resultó un interés de investigación.

Por consiguiente, el objetivo de este trabajo fue analizar la influencia del flujo de batición al calientavinos y de la temperatura del vino a la salida del calientavinos en el flujo másico de aguardiente y de los vapores alcohólicos producidos, así como en la composición del aguardiente.

\section{Materiales y métodos}

\section{Balances de materiales y energía en el sistema de condensadores}

Para llevar a cabo los balances de masa y energía en el sistema de condensadores del tope de la columna de destilación se utilizaron las ecuaciones (1)-(4).

Qabs $=\mathrm{m} * \mathrm{Cp} * \Delta \mathrm{T}$

Qabs=-Qced

$q=-\frac{Q \text { ced }}{\text { vapores }}$

$\mathrm{Hs}=\mathrm{He}+\mathrm{q}$

Donde:

Qabs: calor absorbido $(\mathrm{kJ} / \mathrm{h})$

$\mathrm{m}$ : flujo de la batición $(\mathrm{kg} / \mathrm{h})$

Cp: capacidad calorífica específica de la batición (kJ/ $\mathrm{kgoC})$

$\Delta \mathrm{T}$ : variación de temperatura $\left({ }^{\circ} \mathrm{C}\right)$

Qced: calor cedido ( $\mathrm{kJ} / \mathrm{h}$ )

q: relación entre el calor cedido y el flujo de vapor de entrada al condensador $(\mathrm{kJ} / \mathrm{kg})$

vapores: flujo de vapor de entrada al condensador $(\mathrm{kg} / \mathrm{h})$

He,s: entalpía de entrada y salida respectivamente (kJ/ $\mathrm{kg})$

A partir del conocimiento de las propiedades físicas de la alimentación se utilizó el siguiente procedimiento: Se determinó el calor absorbido mediante la ecuación (1), que es igual al calor cedido (2) si se consideran las pérdidas de calor despreciables. Posteriormente se calculó q mediante la ecuación (3). En el diagrama entalpía-composición para el sistema etanol-agua (Green y Perry, 2008), se halló He. Conocida He, se calculó Hs mediante la ecuación (4) y en el mismo diagrama, con el porcentaje en peso (\% en peso) de los vapores de entrada y Hs se pudo conocer el porcentaje de líquido o de condensado a la salida del condensador. Con la línea de amarre ("tie-line") de ese punto, al cortar las curvas del vapor y el líquido saturado se obtuvo la fracción másica a la salida de la fase vapor y la fase líquida en porcentaje en peso (Rodríguez, 2012).

En el Cuadro 1 se reportan los flujos de las 


\begin{tabular}{lr}
$\begin{array}{l}\text { Cuadro 1. Datos de la columna de } \\
\text { destilación }\end{array}$ \\
\hline Variable (kg/h) & Valor \\
\hline Batición (F) & 40800 \\
Vapor alcohólico (Va) & 5958 \\
Destilado (D) & 1825 \\
Reflujo (R) & 4133 \\
Vinaza (W) & 40670 \\
Vapor (V) & 5828 \\
\hline
\end{tabular}

corrientes asociadas a la columna de destilación requeridos para la realización de los balances de energía en el sistema. El cálculo manual se hizo con el objetivo de verificar el modelo de simulación, que se presenta a continuación:

\section{Simulación en HYSYS}

Para analizar la influencia de algunas variables de operación sobre el flujo de aguardiente y su calidad, se realizó la simulación del proceso de producción de aguardiente en el simulador HYSYS v3.2, que tiene como requerimientos básicos para su instalación: sistema operativo Windows 2000/XP, procesador mínimo Pentium II $400 \mathrm{MHz}$, memoria RAM mínimo 128 MB y espacio libre en disco mínimo $200 \mathrm{MB}$ (HYSYS, 2003).

Se empleó una mezcla de etanol-agua como fluido de trabajo. Para la descripción del comportamiento de la fase de vapor se utilizó el paquete de propiedades IDEAL y para la descripción de la no idealidad de la fase líquida, acorde a la literatura (Marquini et al., 2007) se seleccionó el modelo de actividad de composición local NRTL, sugerido para equilibrios entre el agua y sustancias orgánicas y es el más recomendado para este tipo de sistema en específico.

En la Figura 1 se muestra el diagrama de flujo para la simulación. Se puede observar en este esquema la columna de destilación de aguardiente, el calientavinos (conformado por dos intercambiadores en paralelo; condensador 1 y 2), el condensador primario (dos intercambiadores en paralelo; condensador 3 y 4), el condensador parcial, el condensador total, un enfriador de aguardiente, la torre de enfriamiento, además de otros equipos como los separadores, los cuales muestran la cantidad de vapor sin condensar y a su vez el flujo másico de condensado que se obtiene.

Una vez simulado el esquema de destilación en las condiciones actuales de operación y evaluado el margen de error que introduce esta, se abordaron dos casos de estudio para analizar la influencia del flujo de batición al calientavinos sobre el flujo másico de aguardiente y de la temperatura del vino a la salida del calientavinos sobre el flujo másico y composición del aguardiente y el flujo de los vapores alcohólicos producidos. Los intervalos de flujo y temperatura analizados son de mayor frecuencia de trabajo.

Caso de estudio 1: Cambiar el flujo másico de batición de 30000 hasta $40000 \mathrm{~kg} / \mathrm{h}$. En este primer caso de estudio se analizó cómo influye la variación del

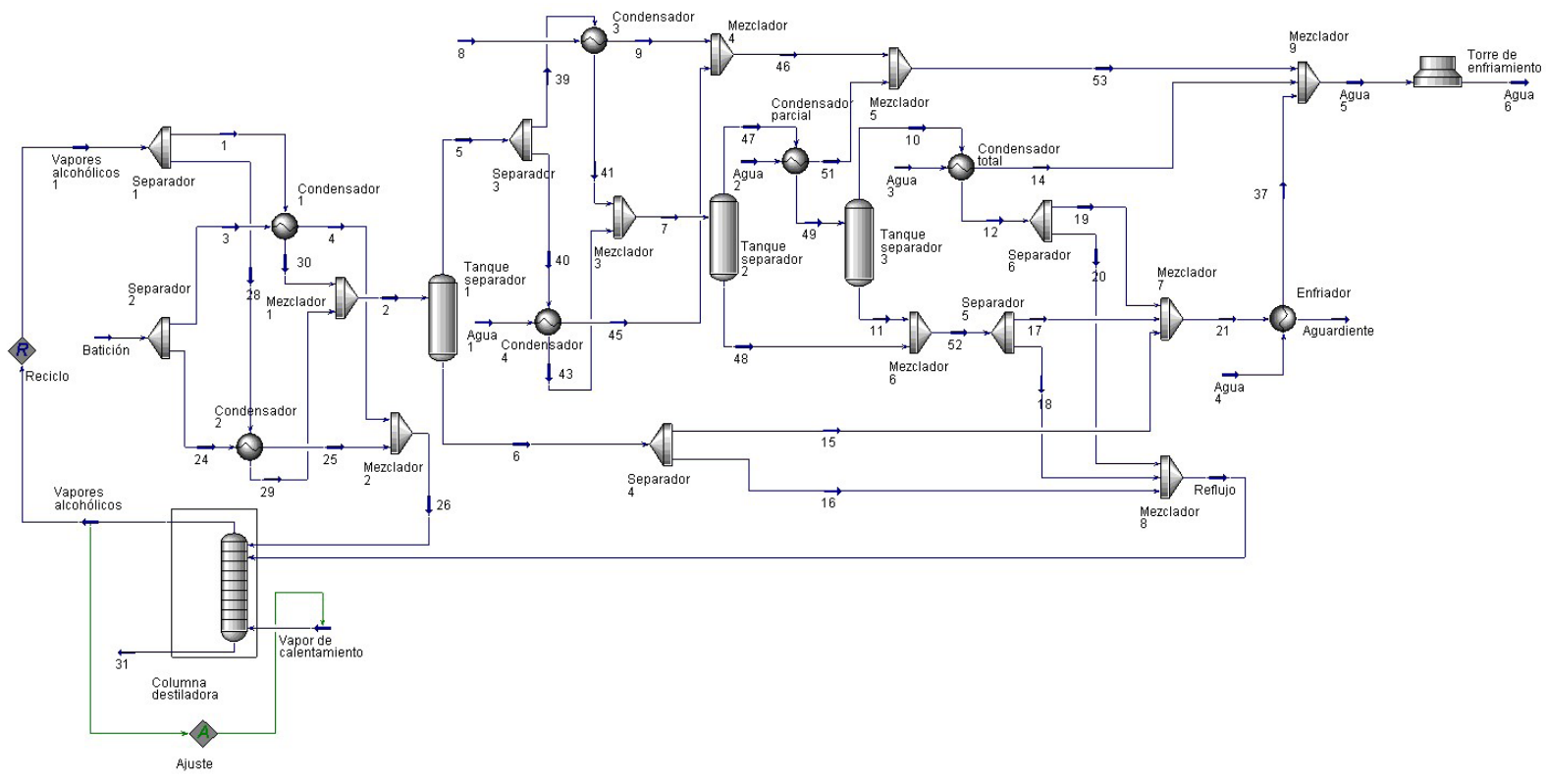

Figura 1. Esquema del trabajo en el simulador de HYSYS 
flujo másico de batición alimentado al calientavinos (variable independiente) con un incremento de 2000 $\mathrm{kg} / \mathrm{h}$, en el flujo másico de aguardiente producido (variable dependiente) y en la fracción másica de etanol (variable dependiente).

Caso de estudio 2: Cambiar la temperatura del vino a la salida del calientavinos (corriente 4) de 70 hasta $90{ }^{\circ} \mathrm{C}$. En este segundo caso de estudio se analizó cómo influye la variación de la temperatura de salida del vino en el calientavinos (variable independiente) con un incremento de $4{ }^{\circ} \mathrm{C}$, sobre el flujo másico de los vapores alcohólicos (corriente vapores alcohólicos) (variable dependiente), el flujo de aguardiente producido (variable dependiente) y la composición de etanol en el aguardiente (variable dependiente). La temperatura máxima está determinada por la temperatura de ebullición de la batición $\left(95^{\circ} \mathrm{C}\right)$.

\section{Balances de materiales y energía en el generador de vapor}

El vapor suministrado a la sección de destilación proviene del área de generación de vapor. Para verificar el desempeño del generador de vapor se evaluó su eficiencia térmica por el método indirecto, según la ecuación (14) y para ello se utilizaron las ecuaciones (5)-(13).

Según, Pérez (2007), para un índice de Bacharach igual a 2, el porcentaje de pérdidas por incombustión mecánica $(\operatorname{Pim}(\%))$ es igual a $0,2 \%$. Por otra parte, $\mathrm{B}$, utilizado en la ecuación (8), es un coeficiente que depende del combustible y se calcula a partir de la ecuación (9). Además, para calderas de petróleo las pérdidas por residuos se consideran despreciables.

Los porcentajes de recuperación sobre la base del combustible quemado y de la pérdida de energía por calor sensible se determinaron a partir de las ecuaciones (16) y (17) respectivamente.

$$
\begin{aligned}
& \propto=\frac{\frac{21}{(1+B)}}{C O_{2}} \\
& V C I=(81 * C+246 * H-26 *(0+S)-6 * W) * 4,186 \\
& P c s(\%)=\frac{K *(T g-T a)}{C O_{2}+C O} \\
& C O(\%)=\frac{\left(21-B * C O_{2}\right)-\left(\mathrm{CO}_{2}+O_{2}\right) \%}{0,605+B} \\
& B=2,85 * \frac{H-0,126 * 0+0,04 * N}{C+0,375 * S} \\
& P i q(\%)=\frac{n_{c o} V C_{c o} * 100}{M_{c} V C I}
\end{aligned}
$$

$$
\begin{aligned}
& P m e(\%)=\sqrt{\frac{100}{M v m a ́ x} * A} \\
& P p(\%)=\frac{P *(h p-h i)}{M c * V C I} \\
& P=\frac{m * D * p p m(a)}{p p m(p)-p p m(a)} \\
& n_{b}=100-(P c s(\%)+P i q(\%)+P m e(\%)+P p(\%)) \\
& P E D=\sum n i * C p i *(T g-T r) \\
& r e c_{1}(\%)=\frac{P E D}{M_{c} * V C I} * 100 \\
& \operatorname{rec}_{2}(\%)=\frac{P E D}{Q_{1}} * 100
\end{aligned}
$$

Donde:

$\alpha$ : coeficiente de exceso del aire

VCI: valor calórico inferior $(\mathrm{kJ} / \mathrm{kg})$

$\mathrm{W}$ : humedad (\% en peso)

$\mathrm{CO}(\%)$ : porcentaje de $\mathrm{CO}$ en los gases de combustión Pcs(\%): porcentaje de pérdidas por calor sensible

Piq(\%): porcentaje de pérdidas por incombustión química

Pme(\%): porcentaje de pérdidas por transferencia de calor al medio exterior

$\mathrm{Pp}(\%)$ : porcentaje de pérdidas por la purga

P: flujo de purga $(\mathrm{kg} / \mathrm{h})$

$\eta b$ : eficiencia térmica del generador de vapor (\%)

PED: potencial energético disponible $(\mathrm{kJ} / \mathrm{h})$

ni: flujo molar de cada especie $(\mathrm{kmol} / \mathrm{h})$

Cpi: capacidad caloríficas medias molares de gases ideales ( $\mathrm{kJ} / \mathrm{kmol} . \mathrm{K})$

rec1(\%): Recuperación sobre la base de combustible quemado (\%)

rec2 (\%): Recuperación sobre la base de la pérdida de energía por calor sensible (\%)

Q1: energía que toman los gases, del calor liberado por el combustible en la combustión

Los datos necesarios para la realización de los balances se presentan en el Cuadro 2 .

El flujo molar de los gases de combustión, así como la temperatura de rocío ácido de los gases de combustión se calcularon a partir del balance de masa en el generador de vapor. 
Cuadro 2. Datos relacionados con la generación de vapor

\begin{tabular}{lr}
\hline Variable & Valor \\
\hline Composición del combustible, peso $(\%)$ & 85,60 \\
$\mathrm{C}$ & 11,40 \\
$\mathrm{H}$ & 3,00 \\
$\mathrm{~S}$ & 1200,00 \\
Masa de combustible $(\mathrm{Mc})(\mathrm{kg} / \mathrm{h})$ & 88,00 \\
Temperatura de entrada del combustible $\left({ }^{\circ} \mathrm{C}\right)$ & 240,00 \\
Temperatura de los gases a la salida del generador $(\mathrm{Tg})\left({ }^{\circ} \mathrm{C}\right)$ & 30,00 \\
Temperatura del aire $(\mathrm{Ta})\left({ }^{\circ} \mathrm{C}\right)$ & 0,02 \\
Humedad másica del aire $\left(\mathrm{Y}_{\mathrm{A}}\right)(\mathrm{kg} \mathrm{H} \mathrm{O} / \mathrm{\textrm {kg }}$ aire$)$ & 17000,00 \\
Producción máxima de vapor $(\mathrm{Mvmáx})(\mathrm{kg} / \mathrm{h})$ & 14000,00 \\
Producción de vapor $(\mathrm{D})$ a $0,9 \mathrm{MPa}$ y $180{ }^{\circ} \mathrm{C}(\mathrm{kg} / \mathrm{h})$ & 1,00 \\
Fracción de agua de reposición $(\mathrm{m})$ & 387,00 \\
Análisis promedio del agua de alimentación $(\mathrm{ppm}(\mathrm{a}))(\mathrm{ppm})$ & 3411,00 \\
Análisis promedio del agua del interior de la caldera $(\mathrm{ppm}(\mathrm{p}))(\mathrm{ppm})$ & \\
Análisis Orsat de los gases de combustión & 12,20 \\
$\mathrm{CO}_{2}(\%)$ & 4,10 \\
$\mathrm{O}_{2}(\%)$ &
\end{tabular}

\section{Resultados y discusión}

$A^{t}$ través del diagrama entalpía-composición para el sistema etanol-agua (Green y Perry, 2008) y aplicando el procedimiento manual descrito anteriormente se obtuvieron los resultados de los balances de materiales y energía para el sistema de condensadores de tope que se presentan en los Cuadros 3 , 4 y 5, que incluyen los equipos calientavinos, condensador primario, condensador parcial y condensador total.

Cuadro 3. Condiciones de operación en el calientavinos

\begin{tabular}{lr}
\hline Variable & Valor \\
\hline Presión de operación de los condensadores $1 \mathrm{y} 2(\mathrm{kPa})$ & 101,30 \\
Temperatura de entrada de los vapores alcohólicos $\left({ }^{\circ} \mathrm{C}\right)$ & 93,70 \\
Fracción másica de etanol en vapores alcohólicos (adimensional) & 0,52 \\
Porcentaje de pérdidas de calor $(\%)$ & 0,00 \\
Temperatura del vino a la entrada $\left({ }^{\circ} \mathrm{C}\right)$ & 34,00 \\
Temperatura del vino a la salida $\left({ }^{\circ} \mathrm{C}\right)$ & 80,00 \\
Entalpía del vino a la entrada $(\mathrm{kJ} / \mathrm{kg})$ & 1838,60 \\
Entalpía del vino a la salida $(\mathrm{kJ} / \mathrm{kg})$ & 603,75 \\
Porcentaje de líquido $(\%)$ & $70-75$ \\
Fracción másica del líquido $(\mathrm{adimensional})$ & 0,43 \\
Fracción másica del vapor $(\mathrm{adimensional})$ & 0,75 \\
Flujo másico de condensado $(\mathrm{kg} / \mathrm{h})$ & 4051,44 \\
Flujo másico de vapor $(\mathrm{kg} / \mathrm{h})$ & 1906,56 \\
\hline
\end{tabular}




\section{Cuadro 4. Condiciones de operación en el condensador primario}

\begin{tabular}{lr}
\hline Variable & Valor \\
\hline Presión de operación de los condensadores 3 y $4(\mathrm{kPa})$ & 101,30 \\
Flujo másico de vapores alcohólicos a condensar $(\mathrm{kg} / \mathrm{h})$ & 1906,56 \\
Temperatura de entrada de los vapores alcohólicos $\left({ }^{\circ} \mathrm{C}\right)$ & 83,06 \\
Fracción másica de etanol en vapores alcohólicos (adimensional) & 0,75 \\
Porcentaje de pérdidas de calor $(\%)$ & 0,00 \\
Temperatura del agua de enfriamiento a la entrada $\left({ }^{\circ} \mathrm{C}\right)$ & 32,00 \\
Temperatura del agua de enfriamiento a la salida $\left({ }^{\circ} \mathrm{C}\right)$ & 78,00 \\
Flujo másico de agua de enfriamiento $(\mathrm{kg} / \mathrm{h})$ & 6000,00 \\
Entalpía del agua de enfriamiento a la entrada $(\mathrm{kJ} / \mathrm{kg})$ & 1466,27 \\
Entalpía del agua de enfriamiento a la salida $(\mathrm{kJ} / \mathrm{kg})$ & 858,81 \\
Porcentaje de líquido $(\%)$ & 46,00 \\
Fracción másica del líquido $($ adimensional $)$ & 0,66 \\
Fracción másica del vapor $($ adimensional) & 0,83 \\
Flujo másico de condensado $(\mathrm{kg} / \mathrm{h})$ & 877,01 \\
Flujo másico de vapor $(\mathrm{kg} / \mathrm{h})$ & 1029,54 \\
\hline
\end{tabular}

Cuadro 5. Condiciones de operación en los condensadores parcial y total

\begin{tabular}{lrr}
\hline Variable & $\begin{array}{c}\text { Condensador } \\
\text { parcial }\end{array}$ & $\begin{array}{c}\text { Condensador } \\
\text { total }\end{array}$ \\
\hline Presión de operación del condensador $(\mathrm{kPa})$ & 101,30 & 101,30 \\
Flujo másico de vapores alcohólicos a condensar $(\mathrm{kg} / \mathrm{h})$ & 1029,54 & 25,74 \\
Temperatura de entrada de los vapores alcohólicos $\left({ }^{\circ} \mathrm{C}\right)$ & 80,17 & 78,78 \\
Fracción másica de etanol en vapores alcohólicos $($ adimensional) & 0,83 & 0,88 \\
Porcentaje de pérdidas de calor $(\%)$ & 0,00 & 0,00 \\
Temperatura del agua de enfriamiento a la entrada $\left({ }^{\circ} \mathrm{C}\right)$ & 32,00 & 32,00 \\
Temperatura del agua de enfriamiento a la salida $\left({ }^{\circ} \mathrm{C}\right)$ & 76,00 & 52,00 \\
Flujo másico de agua de enfriamiento $(\mathrm{kg} / \mathrm{h})$ & 6000,00 & 320,00 \\
Entalpía del agua de enfriamiento a la entrada $(\mathrm{kJ} / \mathrm{kg})$ & 1338,26 & 1268,44 \\
Entalpía del agua de enfriamiento a la salida $(\mathrm{kJ} / \mathrm{kg})$ & 264,86 & 227,57 \\
Porcentaje de líquido $(\%)$ & 97,50 & 100,00 \\
Fracción másica del líquido $($ adimensional) & 0,82 & - \\
Fracción másica del vapor $($ adimensional) & 0,88 & - \\
Flujo másico de condensado $(\mathrm{kg} / \mathrm{h})$ & 1003,80 & - \\
Flujo másico de vapor & 25,74 & - \\
\hline
\end{tabular}


Cuadro 6. Comparación entre los parámetros de operación calculados manualmente y mediante la simulación del sistema

\begin{tabular}{|c|c|c|c|}
\hline Parámetro & HYSYS & Manual & $\begin{array}{c}\text { Error } \\
\text { relativo } \\
(\%)\end{array}$ \\
\hline Flujo másico de vapor a la salida del calientavinos $(\mathrm{kg} / \mathrm{h})$ & 1884,20 & 1906,60 & 1,20 \\
\hline Flujo másico de vapor a la salida del condensador primario $(\mathrm{kg} / \mathrm{h})$ & 1024,40 & 1029,50 & 0,50 \\
\hline Flujo másico de condensado en el condensador primario $(\mathrm{kg} / \mathrm{h})$ & 859,80 & 877,00 & 2,00 \\
\hline Flujo másico de vapor a la salida del condensador parcial $(\mathrm{kg} / \mathrm{h})$ & 24,60 & 25,70 & 4,50 \\
\hline Flujo másico de condensado en el condensador parcial $(\mathrm{kg} / \mathrm{h})$ & 999,80 & 1003,80 & 0,40 \\
\hline \multirow[t]{2}{*}{ Flujo másico de condensado en el condensador total $(\mathrm{kg} / \mathrm{h})$} & 24,60 & 25,71 & 4,50 \\
\hline & \multicolumn{2}{|r|}{ Promedio } & 2,20 \\
\hline Fracción másica de etanol en el vapor de salida del calientavinos & 0,74 & 0,75 & 1,20 \\
\hline Fracción másica de etanol en el vapor de salida del condensador primario & 0,81 & 0,83 & 1,92 \\
\hline Fracción másica de etanol en el vapor de salida del condensador parcial & 0,87 & 0,88 & 0,91 \\
\hline Fracción másica de etanol en el líquido a la salida del condensador parcial & 0,81 & 0,82 & 0,85 \\
\hline \multirow[t]{2}{*}{ Fracción másica de etanol en el vapor de salida del condensador total } & 0,86 & 0,88 & 0,91 \\
\hline & \multicolumn{2}{|r|}{ Promedio } & 1,16 \\
\hline Temperatura del condensado a la salida del calientavinos $\left({ }^{\circ} \mathrm{C}\right)$ & 83,37 & 83,06 & 0,37 \\
\hline Temperatura del condensado a la salida del condensador primario $\left({ }^{\circ} \mathrm{C}\right)$ & 80,29 & 80,17 & 0,15 \\
\hline Temperatura del condensado a la salida del condensador parcial $\left({ }^{\circ} \mathrm{C}\right)$ & 78,89 & 78,89 & 0,00 \\
\hline \multirow[t]{2}{*}{ Temperatura del condensado a la salida del condensador total $\left({ }^{\circ} \mathrm{C}\right)$} & 78,45 & 78,40 & 0,06 \\
\hline & \multicolumn{2}{|r|}{ Promedio } & 0,15 \\
\hline
\end{tabular}

En el Cuadro 6 se presenta la comparación de los principales resultados obtenidos con el simulador HYSYS y los cálculos manuales realizados, así como el error relativo (\%) para cada parámetro. La comparación de los resultados obtenidos, demuestra la posibilidad de la utilización del esquema de simulación realizado para el análisis de alternativas en el proceso, puesto que los valores arrojados por este simulador son similares a los obtenidos por los cálculos manuales desarrollados, siendo el error relativo promedio menor de un 5\%.

En el Cuadro 7 se reflejan los resultados del caso de estudio 1 . Se puede apreciar que al aumentar el flujo másico de batición en un 33\% aproximadamente, se logra un aumento del flujo másico de aguardiente de
$9,38 \%$, lo cual resulta beneficioso, pues incrementa el volumen de producción. Este incremento no es lineal, siendo mayor a medida que el flujo de batición aumenta. Por otra parte, el aguardiente incrementa el contenido de etanol a medida que aumenta el flujo másico de batición. Por consiguiente, resulta conveniente trabajar al mayor valor posible del intervalo.

En el Cuadro 8 se refleja que cuando aumenta la temperatura del vino a la salida del calientavinos en un $29 \%$ aproximadamente, el flujo másico de vapores alcohólicos aumenta en un $18,1 \%$. Esto es conveniente ya que la causa principal de la incrustación exagerada y en corto tiempo en los equipos de intercambio de calor es la alcoholización de la batición dentro de la columna

Cuadro 7. Variación del flujo másico de aguardiente y de su composición con el aumento en el flujo másico de batición (Caso de estudio 1)

\begin{tabular}{lccccccc}
\hline \multicolumn{1}{c}{ Variables } & Paso 1 Paso 2 & Paso 3 & Paso 4 & Paso 5 & Paso 6 \\
\hline Flujo másico de batición $(\mathrm{kg} / \mathrm{h})$ & 30000 & 32000 & 34000 & 36000 & 38000 & 40000 \\
Flujo másico de aguardiente $(\mathrm{kg} / \mathrm{h})$ & 1673 & 1690 & 1713 & 1741 & 1780 & 1830 \\
Fracción molar de etanol en el aguardiente & 0,622 & 0,633 & 0,648 & 0,664 & 0,686 & 0,711 \\
\hline
\end{tabular}


Cuadro 8. Variación del flujo másico de vapores alcohólicos, flujo y composición del aguardiente con la temperatura del vino a la salida del calientavinos (Caso de estudio 2)

\begin{tabular}{|c|c|c|c|c|c|c|}
\hline Variables & $\begin{array}{c}\text { Paso } \\
1\end{array}$ & $\begin{array}{c}\text { Paso } \\
2\end{array}$ & $\begin{array}{c}\text { Paso } \\
\mathbf{3}\end{array}$ & $\begin{array}{c}\text { Paso } \\
4\end{array}$ & $\begin{array}{c}\text { Paso } \\
5\end{array}$ & $\begin{array}{c}\text { Paso } \\
6\end{array}$ \\
\hline Temperatura del vino a la salida del calientavinos (corriente 4$)\left({ }^{\circ} \mathrm{C}\right.$ ) & 70 & 74 & 78 & 82 & 86 & 90 \\
\hline Flujo másico de vapores alcohólicos $(\mathrm{kg} / \mathrm{h})$ & 5,766 & 5,981 & 6,191 & 6,398 & 6,604 & 6,810 \\
\hline Flujo másico de aguardiente $(\mathrm{kg} / \mathrm{h})$ & 1,835 & 1,844 & 1,852 & 1,859 & 1,866 & 1,872 \\
\hline Fracción másica de etanol en el aguardiente & 0,719 & 0,721 & 0,722 & 0,722 & 0,723 & 0,724 \\
\hline
\end{tabular}

destiladora y es por ello que resultará siempre favorable precalentar la batición antes de entrar a la columna hasta valores cercanos a su temperatura de ebullición, $95^{\circ} \mathrm{C}$ aproximadamente. Del cuadro 8 se aprecia que la producción de aguardiente aumenta en un $2 \%$ por esta modificación y que el aguardiente se enriquece en su contenido de etanol a medida que la temperatura aumenta.

Por consiguiente, resulta adecuado proponer como condiciones de operación un flujo másico de batición de $40000 \mathrm{~kg} / \mathrm{h}$ y calentar el vino en el calientavinos hasta $90^{\circ} \mathrm{C}$. Con estas condiciones, de acuerdo a la simulación

Cuadro 9. Balance de energía en el generador de vapor

\begin{tabular}{|c|c|c|}
\hline Parámetro & Valor & $\begin{array}{c}\text { Ecuación de cálculo } \\
\text { o referencia } \\
\end{array}$ \\
\hline Valor calórico inferior del combustible (VCI) (kJ/kg) & 40437,00 & 6 \\
\hline Entalpía del líquido saturado a $0.9 \mathrm{MPa}(\mathrm{hp})(\mathrm{kJ} / \mathrm{kg})$ & 741,83 & (Keenan, 1988) \\
\hline Entalpía del agua de alimentación a 80 (hl) (kJ/kg) & 334,91 & (Keenan, 1988) \\
\hline Calor de combustión del $\mathrm{CO}\left(\mathrm{V}_{\mathrm{CO}}\right)(\mathrm{kJ} / \mathrm{kmol})$ & 282989,40 & (HYSYS, 2003) \\
\hline Entalpía del vapor a $0.9 \mathrm{MPa}$ y $180^{\circ} \mathrm{C}\left(\mathrm{h}_{\mathrm{v}}\right)(\mathrm{kJ} / \mathrm{kg})$ & 22785,50 & (Keenan, 1988) \\
\hline Temperatura de rocío ácido de los gases $(\operatorname{Tr})\left({ }^{\circ} \mathrm{C}\right)$ & 200,00 & \\
\hline \multicolumn{3}{|l|}{ Flujo molar de cada especie en los gases de combustión ( $(\mathrm{kmol} / \mathrm{h})$} \\
\hline $\mathrm{CO}_{2}$ & 84,62 & \\
\hline $\mathrm{CO}$ & 0,97 & \\
\hline $\mathrm{O}_{2}$ & 28,44 & \\
\hline $\mathrm{N}_{2}$ & 579,64 & \\
\hline $\mathrm{H}_{2} \mathrm{O}$ & 8,19 & \\
\hline Coeficiente de exceso del aire $(\alpha)$ & 1,25 & 5 \\
\hline Coeficiente B & 0,37 & 9 \\
\hline Flujo de purga $(\mathrm{kg} / \mathrm{h})$ & 1791,60 & 13 \\
\hline Pérdidas por calor sensible de los gases de combustión (\%Pcs) & 9,96 & 7 \\
\hline Pérdidas por incombustión química (\%Piq) & 0,56 & 10 \\
\hline Pérdidas por incombustión mecánica (\%Pim) & 0,20 & (Pérez, 2007) \\
\hline Pérdidas por transferencia de calor al medio exterior (\%Pme) & 2,91 & 11 \\
\hline Pérdidas por las purgas $(\% \mathrm{Pp})$ & 1,51 & 12 \\
\hline Eficiencia (\%) & 84,86 & 14 \\
\hline Potencial energético disponible (PED) (kJ/h) & 983014,00 & 15 \\
\hline Recuperación sobre la base de combustible quemado $\left(\%\right.$ rec $\left._{1}\right)(\%)$ & 2,03 & 16 \\
\hline Recuperación sobre la base de la pérdida de energía por calor sensible $\left(\% \mathrm{rec}_{2}\right)(\%)$ & 19,05 & 17 \\
\hline
\end{tabular}


realizada se obtendrían $1872 \mathrm{~kg} / \mathrm{h}$ de aguardiente de fracción molar de etanol 0,7235. Se consumirían 5828 $\mathrm{kg} / \mathrm{h}$ de vapor en la columna destiladora y $24340 \mathrm{~kg} / \mathrm{h}$ de agua de enfriamiento en los condensadores.

El vapor que alimenta a la columna destiladora proviene del área de generación de vapor, principal consumidor de petróleo de la instalación. La evaluación del desempeño del generador de vapor permitió conocer si existen posibilidades de ahorro de este combustible. El resultado de la evaluación energética del generador de vapor se presenta en el Cuadro 9, donde se observa que las mayores pérdidas se producen por el calor sensible de los gases de combustión. Este valor depende de la temperatura de los gases de combustión y del coeficiente de exceso de aire $(\alpha)$. En calderas petroleras se recomienda que el exceso de aire no exceda el $20 \%$; en este caso se aprecia un ligero incremento del valor, lo que puede ocasionar una disminución de la temperatura de llama necesitándose un mayor consumo de combustible y mayores temperaturas en los gases de combustión. Es por esto que se necesita mantener limpias las superficies de transferencia de calor y una correcta hermeticidad; además se recomienda controlar que no existan deflectores de gases defectuosos.

De acuerdo a los resultados obtenidos existe posibilidad de recuperación de energía, principalmente a partir de la utilización del calor sensible de los gases de combustión $(983014 \mathrm{~kJ} / \mathrm{h}$ ), con lo que se pudieran adicionar superficies recuperadoras para el calentamiento del combustible, del aire o del agua de alimentación. Esto permitiría recuperar hasta el 19,05\% de la energía que hoy no se utiliza, lo que en términos de combustible no quemado representa $24,34 \mathrm{~kg} / \mathrm{h}$ de combustible menos o sea un consumo $2,03 \%$ menor.

\section{Conclusiones}

Se obtuvo el esquema de simulación de la columna destiladora y el sistema calientavinoscondensadores utilizando el simulador comercial HYSYS 3.2, para su uso en el análisis de alternativas en el proceso. Se concluyó que el incremento del flujo másico de batición y de la temperatura del vino a la salida de la batición permite aumentar la producción de aguardiente, así como su contenido de etanol. Las mejores condiciones de trabajo se alcanzaron para el flujo de batición de $40000 \mathrm{~kg} / \mathrm{h}$ y una temperatura a la salida del calientavinos de $90{ }^{\circ} \mathrm{C}$. Adicionalmente, la evaluación del sistema de generación de vapor arrojó que es posible la recuperación del 19\% de la energía que se emite a la atmósfera como calor sensible de los gases de combustión.

\section{Refrencia Bibliográfica}

Blanco, J. 2011. Automatización y control regulatorio de una columna de destilación extractiva a nivel planta piloto para la producción de etanol anhidro. Proyecto de grado presentado como requisito parcial para optar al título de Magíster en Automatización Industrial. Facultad de Ingeniería. Universidad Nacional de Colombia. Bogotá. Colombia. 65 p. Recuperado 15 de mayo de 2017, a partir: http://bdigital.unal.edu. co/5094/1/299977.2011_pte._1.pdf

Cardona, C., Sánchez, O., Montoya, M., Quintero, JA. 2005. Simulación de los procesos de obtención de etanol a partir de caña de azúcar y maíz. Scientia et Technica XI (28): 187-192.

Duro, C., Morilla, G. 2016. Modelado y simulación de columnas binarias de destilación con control de inventario. Recuperado 27 de julio de 2017, a partir: http://www.dia. uned.es/ fmorilla/WebFMorillaJulio2013/ Ultimaspublicaciones/2001CDMMSS2001.pdf.

Green, D., Perry, R. 2008. Perry's Chemical Engineers' Handbook. Editorial McGraw Hill. 8va ed.

Fernández, EH. 2016. Tecnología de bebidas. Vol 1. Bebidas alcohólicas. Coordenador Waldemar Gastoni Venturini Filho. Parte II. Bebidas destiladas Capítulo 22. Rum. Editorial Edgar Blucher. Sao Paulo. Brasil. Págs 317-329.

HYSYS. 2003. Version 3.2 Hyprotech Ltd. Calgary, Canada.

Keenan, J., Keyes, F., Hill, P., Moore, J. 1988. Steam Tables. Editorial Revolucionaria. Cuba. 156 p.

Oliveira, T., Batista, F., Bernadate, M., Batista, J. 2016. Producción de aguardiente utilizando extracto de alfa ácidos del lúpulo en el control biocida del proceso fermentativo. Centro Azúcar 43(1): 1824.

Marquini, MF., Mariani, DC., Meirelles, AJA., dos Santos, OAA., Jorge, LMM. 2007. Simulação e análise de um sistema industrial de colunas de destilação de etanol. Acta Sci. Technol. 29(1): 2328.

Pérez, O. 2011. Modelación, simulación y análisis con fines energéticos de destilerías de etanol hidratado. Tesis presentada en opción al grado científico de doctor en ciencias técnicas. Facultad de Ingeniería Química. Instituto Superior Politécnico José Antonio Echeverría. La Habana. Cuba. 247 p. Recuperado 20 de junio de 2017, a partir: http://beduniv.reduniv.edu.cu/index. php?page $=13 \& \mathrm{id}=431 \& \mathrm{db}=1$

Pérez, O., Zumalacárregui, L., Gozá, O. 2010. Simplificaciones en el cálculo de columnas de 
destilación alcohólica. Información Tecnológica 21(6): 103-112.

Pérez, Y. 2007. Manual para la gestión eficiente de la energía en la industria alimentaria. Viena: Editorial Federico Engels. pp 97-127.

Rodríguez, Z. 2012. Nuevo calientavinos para el sistema de producción de aguardiente. Tesis presentada en opción al título de ingeniera química. Facultad de Ingeniería Química. Instituto Superior Politécnico José Antonio Echeverría. La Habana. Cuba. 99 p. Recuperado 5 de enero de 2017, a partir: http://tesis.cujae.edu.cu/ bitstream/handle/123456789/3951/rodriguez_cabrera zuanchyt.pdf? sequence $=1 \&$ isAllowed $=y$ 